\title{
MadAlegria: a valorização de estratégias de humanização na formação do profissional de saúde
}

\author{
MadAlegria: the value of humanization strategies in the training \\ of health professionals
}

\author{
Key Fujisaki Utsunomiya ${ }^{1}$, Maria Aparecida Basile², Thiago Edi Landim Lopes ${ }^{3}$, \\ Leila Tiemi Okajima ${ }^{3}$, Elizabeth Alves Gonçalves Ferreira ${ }^{4}$
}

\begin{abstract}
Utsunomiya KF, Basile MA, Lopes TEL, Okajima LT, Ferreira EAG. MadAlegria: a valorização de estratégias de humanização na formação do profissional de saúde / MadAlegria: the value of humanization strategies in the training of health professionals. Rev Med (São Paulo). 2015 abr.-jun.;94(2):87-93.

RESUMO: O MadAlegria é um projeto de extensão universitária da Faculdade de Medicina da Universidade de São Paulo, elaborado com a finalidade de aprimorar a habilidade de seus voluntários de criar vínculo profissional com o paciente e melhorar o acolhimento no sistema de saúde. O MadAlegria tem como objetivo amenizar o ambiente hospitalar e outros cenários de prática de saúde, promover o trabalho em equipe multidisciplinar e sensibilizar os estudantes para a importância da humanização na relação entre os profissionais da saúde

de São Paulo (ICESP) foram mantidos, e acrescidos de atendimentos no Instituto Central do Hospital das Clínicas da FMUSP (ICHC), onde o MadAlegria participa da Rede Humaniza FMUSPHC. A manutenção do trabalho voluntário pelos participantes formados no projeto tem sido um grande desafio. A experiência vivida em um projeto de extensão universitária como o MadAlegria é intensa e há relatos de alunos que demonstram que essa vivência influencia o perfil profissional, tornando mais humanizado o cuidado com o outro.
\end{abstract} e o paciente, incentivando assim o exercício da cidadania por meio de recursos lúdicos da figura do Palhaço e do Contador de Histórias em hospital. O projeto foi iniciado em 2010 e desde 2011 foram formados 134 Palhaços em Hospital e 35 Contadores de Histórias. Com cinco anos de existência o projeto evoluiu com a inclusão de alunos de cursos da USP, além dos da área de saúde, professores, outros profissionais da saúde e usuários do sistema. A Missão, Visão e Valores do MadAlegria foram definidos, bem como houve uma readequação de seu Estatuto. Os atendimentos semanais no Instituto do Câncer do Estado
DESCRITORES: Humanização da assistência; Trabalhadores voluntários de hospital; Terapia pela arte; Educação em saúde; Narração.

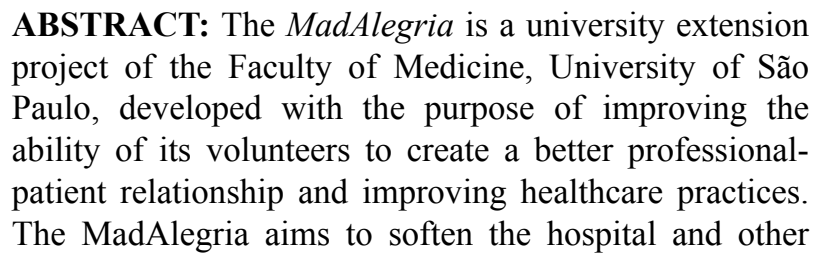

ABSTRACT: The MadAlegria is a university extension project of the Faculty of Medicine, University of São Paulo, developed with the purpose of improving the ability of its volunteers to create a better professionalThe MadAlegria aims to soften the hospital and other

1. Graduanda em Fisioterapia, Faculdade de Medicina da Universidade de São Paulo (FMUSP).

2. Professora Doutora da Faculdade de Medicina da Universidade de São Paulo (FMUSP).

3. Graduandos em Medicina, Faculdade de Medicina da Universidade de São Paulo (FMUSP).

4. Professora Doutora do Curso de Fisioterapia da Faculdade de Medicina da Universidade de São Paulo (FMUSP).

Endereço para correspondência: MadAlegria. Depto. de Moléstias Infecciosas e Parasitárias da FMUSP. Av. Dr. Arnaldo, 455 - sala 2308. Cerqueira César, São Paulo, SP. CEP: 01246-903. E-mail: mad.alegria@gmail.com 
health care practice settings, promoting multidisciplinary team work and sensitize students to the importance of humanizing the relationship between health professionals and patients, and encouraging active citizenship through playful features such as clown and storyteller in hospital. The project was initiated in 2010 and since 2011, were formed 134 Clowns in hospital and 35 Storytellers. With five years of existence, the project has evolved beyond the healthcare students, students from other courses at USP, teachers, other health professionals and system users. The Mission, Vision, Values and Statute of MadAlegria were settled. The weekly interventions were held in ICESP

\section{INTRODUÇÃO}

$\mathrm{N}$ o Brasil, o termo humanização começou a ser empregado nos anos 90, quando usuários e profissionais da saúde reivindicaram melhorias no Sistema Único de Saúde (SUS), principalmente para atender a necessidade de grupos específicos, como o parto humanizado para as gestantes e melhorias no atendimento de bebês internados em UTI. O Plano Nacional de Humanização da Assistência Hospitalar (PNHAH), que durou de 2000 a 2002, identificou que "na avaliação do público, a forma do atendimento, a capacidade demonstrada pelos profissionais de saúde para compreender suas demandas e suas expectativas são fatores que chegam a ser mais valorizados que a falta de médicos, a falta de espaço nos hospitais e a falta de medicamentos".

Em 2003, foi inserido no SUS a Política de Humanização da Assistência à Saúde (PHAS), a fim de qualificar todo o serviço de saúde no país. As ações propostas por essa política no ambiente hospitalar tinham como princípios fundamentais o acolhimento e atendimento dos usuários, a melhoria nas condições de trabalho dos profissionais e o aprimoramento das lógicas de gestão e gerência.

A transformação do sistema de saúde exige mudanças, não apenas de gestão, mas também de seus usuários e trabalhadores, que são os principais elementos do ambiente hospitalar.

A humanização engloba tratamento e cuidado digno, solidário e acolhedor por parte do profissional de saúde, além de uma postura ética ${ }^{2}$. Humanizar é uma estratégia de interferência no processo de atenção à saúde, com maior foco na interação qualificada entre os profissionais e entre os profissionais e usuários do sistema, articulando os avanços tecnológicos com uma comunicação adequada. Nessa estratégia, a comunicação é essencial, ou seja, saber falar e ouvir, reconhecendo a importância da liberdade de expressão cultural e emocional do outro para melhorar a qualidade de atendimento, trabalho e gestão ${ }^{3}$.

O movimento de humanização hospitalar tem foco and were increased with attendance at the Hospital das Clínicas, where MadAlegria is part of Rede Humaniza FMUSPHC. The maintenance of volunteer work by graduate student on the project has been a great challenge. The experience in a university extension project as MadAlegria is enthusiastic and there are reports of students who demonstrate that this experience influences the professional profile, making it more humanized care for the other.

KEYWORDS: Humanization assistance; Hospital volunteers; Art therapy, Health education; Narration.

no processo de educação e treinamento dos profissionais de saúde, bem como em intervenções que façam a experiência da hospitalização ser mais agradável para o paciente ${ }^{4}$. Com esse intuito, estudantes da área da saúde da Universidade de São Paulo (USP), idealizaram um grupo com uma perspectiva humanística para a própria formação e para prestar serviço à comunidade por meio de atendimentos nos hospitais: nascia assim o MadAlegria!

O MadAlegria tem como objetivo amenizar o ambiente hospitalar e outros cenários de prática de saúde, promover o trabalho em equipe multidisciplinar e acima de tudo sensibilizar os estudantes para a importância da humanização na relação entre os profissionais da saúde e o paciente, incentivando o exercício da cidadania. A intenção é dar ao estudante a oportunidade de ampliar a sua visão em relação ao paciente, sensibilizando-o sobre a importância da humanização na área da saúde e a responsabilidade que se tem frente ao sofrimento do outro, a fim de formar melhores profissionais de saúde, com maior engajamento e responsabilidade no exercício profissional ${ }^{5,6}$.

\section{OBJETIVO}

O objetivo deste artigo é relatar a experiência do Projeto de Humanização MadAlegria direcionado à formação do profissional de saúde e baseado nas figuras do Palhaço em Hospital e do Contador de Histórias.

\section{HISTÓRIA DO MadAlegria}

Em agosto de 2010, os estudantes dos cursos de Enfermagem, Fisioterapia, Fonoaudiologia, Medicina, Nutrição e Terapia Ocupacional daUSP se reuniram para dar início ao Projeto Multidisciplinar de Extensão Acadêmica MadAlegria. O Projeto recebeu apoio institucional da FMUSP e a adesão de docentes dos mesmos cursos. O MadAlegria tinha como meta desenvolver atividades culturais, científicas e educacionais com enfoque na 
humanização do relacionamento com os pacientes e com a equipe de saúde.

Em 2011 ocorreu a formatura da primeira turma do MadAlegria de Palhaços em Hospital e em 2012 houve reformulação do treinamento com parceira estabelecida com a Organização Não Governamental (ONG) Doutores da Alegria, que passou a administrar o curso de palhaços em hospital, e o início da formação em Contadores de Histórias com a equipe da arte-educadora Débora Kikuti. Em 2013, o projeto abriu vagas para a comunidade externa e, atualmente, conta com a participação de professores, outros profissionais da saúde e de usuários do sistema. De 2011 a 2015 foram formados 134 Palhaços em Hospital e 35 Contadores de Histórias, que realizam atendimento semanal nas enfermarias do ICESP e do ICHC no período letivo.

\section{Organização do MadAlegria}

O Projeto é constituído de uma coordenação discente e uma coordenação docente, ambas de caráter multiprofissional. Anualmente, ocorre o Curso Introdutório que tem duração de dois dias, ocasião em que há a apresentação do projeto para os interessados. Após este curso, há um processo seletivo para 30 vagas de formação de Palhaços em Hospital e 20 vagas para Contadores de Histórias.

Curso de Formação para Palhaço em Hospital: Este curso de formação não tem como meta formar atores e sim desenvolver habilidades de comunicação, que facilitem a abordagem lúdica do paciente. Nas aulas são vivenciadas técnicas para treino da atenção, desinibição, cumplicidade entre os colegas e abordagem dos pacientes. A música, o improviso, a capacidade de observar o outro, e rapidamente se conectar ao seu espaço e momento, são estratégias amplamente discutidas e vivenciadas no treinamento ${ }^{5}$. No decorrer do curso, os estudantes decidem sobre o nome e características do seu palhaço, salientando que o incentivo é para que não seja muito exuberante ou intensa a caracterização, e sim que seja somente um jeito do paciente identificar que há um palhaço, mas com a discrição que o ambiente hospitalar exige. Todos os palhaços utilizam avental específico do MadAlegria e nas aulas há discussão sobre as sensações dos participantes, bem como as facilidades e dificuldades para a abordagem dos pacientes no hospital. A formação tem duração de um ano, com aulas semanais noturnas de três horas de duração e estágio supervisionado/atendimentos realizados semanalmente no ICHC e no ICESP.

Curso de Formação para Contador de Histórias: A utilização do Contador de Histórias como um recurso comunicativo e de relacionamento leva em conta que ao contar histórias, não ocorre apenas a leitura ou a contação da história, mas também a escuta. A narrativa proporciona uma relação simétrica e privilegiada entre contador e ouvinte, na qual pode-se criar um cenário confortável para a interação entre ambos. A narrativa é trazida para a clínica como uma ferramenta que pode facilitar o entendimento e a leitura do significado do processo de adoecimento, para que o profissional de saúde possa compreender melhor a história do paciente e, desta forma, entender de maneira global o estado clinico de cada sujeito $^{7,8}$. Nas aulas são abordados conteúdos como tipos de histórias, contextualização da história e outros temas relacionados à oralidade, linguagens, repertório e uso de recursos auxiliares, como livros, música e manipulação de bonecos. Todos os Contadores utilizam também avental específico do MadAlegria e a formação tem duração de um ano, com aulas noturnas semanais de três horas de duração, sendo que o treinamento prático supervisionado e os atendimentos são realizados quinzenalmente no ICHC.

\section{Trajetória do MadAlegria}

Em cinco anos houve grande desenvolvimento deste projeto de extensão universitária e o treinamento ofertado tanto para a formação de Palhaço em Hospital, como para Contador de Histórias foi reformulado para melhor adequação aos objetivos propostos. Os atendimentos que eram realizados somente no ICESP, estenderam-se também ao ICHC e, atualmente, o MadAlegria participa do Grupo de Trabalho de Humanização do Voluntariado - Rede Humaniza FMUSPHC. Do ponto de vista científico houve publicação de $\operatorname{artigos}^{5,6}$ e participação em Congressos/Eventos com apresentação de pôsteres, palestras, intervenções e coordenação de oficinas. Neste processo de expansão ficou evidente a necessidade de definir e oficializar a essência do projeto, e para isso foram estabelecidos a Missão, Visão e Valores do MadAlegria.

Missão: Desenvolver habilidades comunicacionais e promover o aprimoramento das relações interpessoais, auxiliando estudantes e profissionais a exercer o cuidado aos usuários do sistema de saúde por meio de estratégias lúdicas, como palhaços em hospital e contadores de histórias.

Visão: Ser uma referência de atividade de cultura e extensão universitária na sensibilização de estudantes e profissionais em saúde para o aprimoramento da empatia, da solidariedade e cidadania nos diferentes cenários de atenção à saúde.

Valores: Compromisso, ética, respeito, empatia, criatividade, pró-atividade e solidariedade.

\section{RESULTADOS}

Para mensurar o sucesso de um projeto de extensão focado na humanização é necessário avaliar os resultados 
objetivos e subjetivos. Na Tabela 1 há a apresentação dos dados referentes à distribuição dos participantes entre os cursos do MadAlegria e na Tabela 2 podem ser observados os dados referentes aos pacientes atendidos no período de 2011 a 2014. A seguir, são apresentados alguns relatos de experiência.

Tabela 1: Distribuição por ano e curso dos participantes selecionados e formados no MadAlegria

\begin{tabular}{lccccc}
\hline & $\mathbf{2 0 1 1}$ & $\mathbf{2 0 1 2}$ & $\mathbf{2 0 1 3}$ & $\mathbf{2 0 1 4}$ & $\mathbf{2 0 1 5}$ \\
Seleção Palhaços & 46 & 51 & 30 & 30 & 30 \\
Palhaços Formados & 38 & 42 & 30 & 25 & - \\
Seleção Contadores & 0 & 20 & 21 & 18 & 20 \\
Contadores Formados & 0 & 10 & 14 & 11 & - \\
\hline
\end{tabular}

Tabela 2: Distribuição por ano do número de pacientes atendidos pelos voluntários do MadAlegria

\begin{tabular}{lcccc}
\hline & $\mathbf{2 0 1 1}$ & $\mathbf{2 0 1 2}$ & $\mathbf{2 0 1 3}$ & $\mathbf{2 0 1 4}$ \\
Palhaços - ICESP & 978 & 541 & 748 & 603 \\
Palhaços - ICHC & 0 & 1053 & 827 & 639 \\
Contadores - ICHC & - & 0 & 120 & 282 \\
\hline
\end{tabular}

\section{Alguns relatos de participantes}

"Como caloura em 2014 estava muito animada com tudo que a faculdade estava me proporcionando naquele primeiro momento e entre todas as apresentações a que mais me chamou a atenção foi a do MadAlegria por ser um projeto de humanização, que poderia me ajudar a ter outra visão diante da minha vida profissional. Com o decorrer do curso pude ver o quão importante é este papel na vida dos pacientes, um trabalho voluntário que te ensina a olhar o indivíduo como um todo e, por consequência, tratando-o além da doença, isto é, se importando com os sentimentos e opiniões dos mesmos; aprendi que o palhaço em meio a tantas coisas obrigatórias da rotina de um hospital é opcional..." (L.N.S., graduanda Fonoaudiologia).

“... Entrava no quarto dos pacientes muitas vezes tomado pelo nervosismo, o medo de desapontar aquela pessoa que depositava expectativas naquele momento de quebra de rotina. Afinal, um grupo de palhaços no horário do Jornal Nacional é um tanto singular..." (F. S., graduando Medicina).

" Cada atendimento é uma experiência única. Entramos no quarto, nos apresentamos e encontramos ali pessoas mergulhadas no sofrimento, nas felicidades momentâneas, na incerteza, na esperança. Algumas precisando de ajuda para escapar dessas emoções, outras para melhor entender e aceitar o que sentem. Explicamos que estamos ali para contar uma história, e logo recebemos uma pequena fração das emoções que contaminam aquelas pessoas... Ocasionalmente, recebemos também um não, o qual é bem vindo, já que esse é o único momento da internação em que a vontade do paciente pode ser respeitada” (A.C.S., graduanda Medicina).
"... dividia os meus dias entre aulas, plantões diurnos e noturnos pelas enfermarias do hospital, mas com um detalhe: um dia da semana aspirante a médico de dia, e palhaço em hospital à noite. Semelhante a um plantão de 12 horas, porém, dessa vez, bem cômico. Em meio ao embaraço de ver os residentes, enfermeiras e pacientes que cumprimentava naquela mesma manhã de trabalho, um pensamento efêmero $e$, às vezes, consolador de que não me reconheceriam com o nariz vermelho vinha à tona. Contudo, se desfazia tão logo era saudado aos risos... E ao sair do quarto vinha a dúvida: Será que fui descoberto?" (F. S., graduando Medicina).

"Estávamos no ICESP eu e mais uma Palhaça. Entramos em um quarto em que os familiares estavam quietos, tristes e a paciente não parecia ter contato com eles. Talvez estivesse dormindo ou somente de olhos fechados. Pedimos autorização para nos aproximarmos do leito e a família gentilmente disse que éramos bem vindos, mas que não adiantava, porque a paciente não se comunicava mais... Como fomos autorizadas, nos aproximamos do leito e com voz baixa e suave nos apresentamos com os nossos nomes de palhaças: Dra. Felícia e Dra. Rosalinda. A minha colega palhaça, sempre fazia uma brincadeira com o seu nome dizendo que era Rosa de família e linda por ela mesma...

Ela falou essa brincadeira e eu disse para a paciente que não aguentava mais ouvir minha colega dizer aquilo e a saída seria fazê-la cantar para parar de falar, mas para isso eu precisava da ajuda da paciente, que ela fizesse esse pedido. Combinamos que se a paciente estivesse nos ouvindo e quisesse ouvir uma música deveria fazer algum sinal. A paciente levantou o polegar e começamos a cantar bem baixinho a música "Alecrim Dourado". Fomos bem até a segunda estrofe e quando ficou evidente que não sabíamos os versos, a paciente começou 
a cantar conosco e nos ensinou a letra. Ela estava bastante debilitada, cantava com dificuldade, mas foi emocionante para nós e para a família vê-la se conectar com a vida pela música naquele instante. Foi um aprendizado para a vida, muito além da sala de aula..." (E.A.G.F., docente Fisioterapia; T.T.G., graduanda Fisioterapia).

“...o paciente revela ao decorrer da visita aquilo que ele mais precisa: uma conversa, uma música ou somente um ouvinte" "Aprendi muito a valorizar os interesses do paciente e buscar meios de violá-los o menos possível". (L.N.S., graduanda Fonoaudiologia).

"Numa noite fria de atendimento supervisionado no ICHC, o trio de palhaços foi levado para conversar com uma paciente de 78 anos, que faria uma grande cirurgia na manhã seguinte e estávamos animados, pois um dos colegas sabia cantar uma linda canção de ninar em japonês, que pensamos ser do agrado da paciente de ascendência oriental... Ela, que estava aninhada no cobertor, ficou entusiasmada com nossa presença, logo se sentou no leito e começou a interagir... No final, propusemos que ela escolhesse a música que mais gostasse e, para nossa surpresa, ela escolheu "Trem das Onze" e cantou conosco!" (M.A.B., docente Medicina).

“... Hoje, porém, olho para trás e não sei dizer se fui eu que consegui ajudar mais os pacientes ou se foram eles que me ensinaram mais" (A.C.S., graduanda Medicina).

\section{DISCUSSÃO}

Em cinco anos de projeto houve expansão do MadAlegria com formação de turmas anuais de palhaços e de contadores de histórias em hospital, inclusão de participantes de diversos cursos, funcionários, professores e usuários, atendimentos em múltiplas alas hospitalares e sua inclusão no Grupo de Trabalho de Voluntariado da Rede Humaniza/ FMUSPHC. E, isto, devido à próatividade e ao compromisso de docentes, discentes e demais participantes, o que é fundamental para a continuidade do projeto.

Aula teórica-prática sobre "Prevenção de Infecção Hospitalar" foi introduzida a todos os participantes, em parceria com a Comissão de Controle de Infecção Hospitalar do ICHC, para treinamento e conscientização sobre a importância da prática de higienização das mãos.

A inclusão no MadAlegria de estudantes de outros cursos, residentes, pós-graduandos, e de profissionais do Complexo HC/FMUSP e externos se deu pela demanda crescente dos interessados em sua proposta, o que demonstra a procura que há por projetos de humanização e trabalho voluntário em hospitais. Até o momento, 13 participantes externos à área da saúde completaram a formação de Palhaços em Hospital e três o curso de Contação de Histórias. Outro aspecto relevante é que aqueles que não são profissionais da saúde trazem para o projeto a visão do usuário e abandonam a postura passiva e dependente dentro da rede de assistência, para colaborar de forma ativa para a melhoria do sistema de saúde.

OCurso de Contação de Histórias, que se iniciou em 2012 como um curso complementar para o atendimento de Palhaços em Hospital, tornou-se um curso independente a partir de 2013, sendo que em 2015 no processo seletivo a demanda para o curso de Contação de Histórias foi a mesma que ocorreu para o curso de Palhaço em Hospital. Em 2013 foram iniciados os atendimentos no ICHC pelos Contadores de Histórias do MadAlegria e em 2014 duplicou o número de atendimentos realizados, conforme é apresentado na Tabela 2. Em 2012, os atendimentos realizados pelos palhaços, que antes ocorriam somente no ICESP, passaram a acontecer também em várias Clínicas no ICHC.

De acordo com os resultados das Tabelas 1 e 2 , nota-se a formação de 134 palhaços e 35 contadores de histórias, com atuação no ICESP e no ICHC. Em 2012, 51 participantes iniciaram a formação com os Drs. da Alegria, sendo 40 candidatos selecionados e 11 da turma de 2011 na qualidade de monitores, sendo que 42 completaram esta formação ( 33 da turma nova e 9 da turma anterior).

Em relação à Tabela 1 , nota-se que em todos os anos o número de formados é inferior ao de selecionados devido à evasão dos alunos por inúmeras razões, como problemas pessoais e familiares, sobrecarga de atividades, inúmeras atividades de extensão concorrentes e desencanto com a exigência na formação/responsabilidade do trabalho voluntário. Este é um aspecto que tem sido motivo de reflexão e preocupação por parte das Diretorias do MadAlegria e dos nossos formadores, o que tem levado a mudanças para aperfeiçoamento do processo seletivo e dos cursos de formação.

A partir de 2012, o número de atendimentos anuais, que em 2011 era de 978, passou a ser de aproximadamente 1500 intervenções, com a incorporação dos atendimentos da Contação de histórias a partir de 2013.

A internação hospitalar para muitos representa um período de vulnerabilidade, uma vez que o paciente sentese angustiado, inseguro, com medo da doença e afastado de sua rotina, sendo frequentemente cuidado pela equipe de saúde de maneira impessoal ${ }^{9}$.

Além de recursos tecnológicos e profissionais competentes, a humanização é essencial para uma experiência hospitalar agradável e obtenção de resultados positivos no tratamento aplicado. $\mathrm{O}$ uso de recursos lúdicos facilita a redução da tensão e do sofrimento da hospitalização, mostrando-se como fator restaurador da saúde, por meio da interação, da empatia, do vínculo, do acolhimento e do cuidado com o próximo, o que é válido não só para crianças, mas também para adultos ${ }^{10}$.

Nas enfermariasé muito comum que os funcionários do hospital e os familiares peçam para serem fotografados ao lado dos palhaços e contadores. Essa atitude simples significa muitas vezes uma novidade na rotina hospitalar 
e um novo assunto a ser conversado entre o paciente e seu acompanhante, e socializado com suas famílias, tema que não é relacionado à doença ou à hospitalização. Estudos demonstram que o impacto da visita de palhaços em hospital ocorre antes do encontro, por antecipação, no momento da interação e após a despedida, tanto para pacientes como para acompanhantes e funcionários. As fotos ou desenhos realizados durante os atendimentos incentivam o efeito tardio da intervenção ${ }^{11}$. Observa-se no relato dos integrantes do MadAlegria que o efeito da interação com os pacientes perdura como experiência importante e que é retomada na memória durante a atuação deles no hospital como profissionais de saúde, e este é o maior objetivo do MadAlegria como estratégia de humanização no ensino.

$\mathrm{O}$ adulto hospitalizado, em geral, tem o desejo de ser mais atuante no seu próprio tratamento, menos dependente da equipe de saúde ${ }^{12}$ e a relação simétrica que o paciente pode experimentar com o Contador de Histórias ou com o Palhaço em Hospital é para o adulto uma oportunidade de se sentir mais protagonista no cenário hospitalar e para o aluno da área da saúde é um treino para incluir o paciente na dinâmica como alguém ativo.

Acredita-se que o humor tem efeitos positivos para o paciente e para o profissional de saúde, contribuindo para a diminuição do estresse e instauração de vínculo ${ }^{13}$. Dessa forma, pode-se dizer que o palhaço cura por meio do riso ${ }^{14}$. Para estudantes da área da saúde, a percepção também é positiva: relatam que as visitas diminuem o estresse do paciente e dos acompanhantes, contribuem para a recuperação dos enfermos e para a formação humanizada do profissional de saúde, considerada essencial, além de auxiliar na prática da multidisciplinaridade ${ }^{15}$.

De modo análogo, a Contação de histórias em hospital é capaz de agir como fator humanizador e terapêutico. Ao ouvir histórias, o paciente coloca-se como protagonista, pois se identifica e é apto a interpretar sua própria realidade sob um novo ponto de vista, vivenciando novas experiências sem sair do lugar. Ao contar histórias, o indivíduo é livre para definir cenários, atores e acontecimentos, que muitas vezes fazem parte de sua própria vida, dando um toque pessoal à fantasia ${ }^{16}$.

Estudos revelam que a participação de estudantes da área da saúde em programas de Contação de histórias melhora suas atitudes em relação a pacientes ${ }^{17}$.
Em contrapartida, a humanização enfrenta obstáculos, já que precisa se estabelecer como um movimento da sociedade brasileira, além de limites estatais, ou seja, redes como o HumanizaSUS necessitam de consolidação além do SUS, como uma verdadeira política pública $^{18}$.

$\mathrm{O}$ que tem sido mais difícil administrar no MadAlegria é a continuidade dos atendimentos pelos voluntários após a finalização dos cursos de formação específicos. A oferta de atividades na Universidade é muito grande e o compromisso com o voluntariado é um exercício de determinação, que nem sempre é fácil de ser cumprido. A convivência com outros grupos de voluntariado nos fez compreender que essa é uma dificuldade pertinente ao universo do voluntariado e que necessita de atenção constante. Algumas atividades como as aulas conjuntas mensais de supervisão dos Doutores da Alegria com todas as turmas e comemorações conjuntas têm como objetivo discutir as intervenções, rever estratégias, acompanhar o desenvolvimento das atividades e fortalecer o vínculo entre os participantes do MadAlegria. Acreditamos que a sensação de pertencimento tende a aumentar a responsabilidade para com a equipe e, em consequência, a aderência ao projeto em comum. Entretanto, temos também casos de estudantes que já finalizaram a graduação e continuam realizando os atendimentos nos hospitais, bem como daqueles que retornaram ao projeto após longo período de intercâmbio no exterior. Estes fatos são indícios que a memória e a vivência obtida com a participação no MadAlegria são intensas, e acompanham os nossos egressos além dos muros da Universidade.

\section{CONCLUSÃo}

O Projeto de Cultura e Extensão MadAlegria tem propiciado que estudantes, profissionais e usuários possam desenvolver suas habilidades comunicacionais, o aprimoramento das relações interpessoais e da empatia por meio de estratégias lúdicas, sensibilizando-os para a humanização do cuidar em saúde e o desenvolvimento da cidadania. E, para tal, tem implementado mudanças para se adequar aos seus objetivos propostos, a fim de manter a qualidade de suas atividades, diminuir a evasão no período de formação e aumentar a adesão ao trabalho voluntário e cidadão.

Agradecimentos: À Pró-Reitoria de Cultura e Extensão da USP, Diretoria da FMUSP, Comissão de Cultura e Extensão FMUSP, Fundação Faculdade de Medicina, ICHC, ICESP, Depto. de Moléstias Infecciosas e Parasitárias da FMUSP, Centro Acadêmico Oswaldo Cruz, Grêmio dos Funcionários da FMUSP (GREMUSP) e Centro de Desenvolvimento de Educação Médica Prof. Eduardo Marcondes (CEDEM) pelo apoio irrestrito ao projeto. Aos participantes do MadAlegria. 


\section{REFERÊNCIAS}

1. Brasil. Ministério da Saúde. Programa Nacional de Humanização da Assistência Hospitalar. Brasília; 2001.

2. Backes DS, Lunardi VL, Lunardi Filho WD. A humanização hospitalar como expressão da ética. Rev Latino-am Enfermagem. 2006;14(1):132-5.

3. Deslandes SF. Análise do discurso oficial sobre humanização da assistência hospitalar. Ciênc Saúde Colet. 2004;9(1):7-13.

4. Mota RA, Martins CGM, Véras RM. Papel dos profissionais de saúde na política de humanização hospitalar. Psicol Estudo. 2006;11(2):323-30.

5. Utsunomiya KF, Ferreira EAG, Oliveira AM, Arai HT, Basile MA. MadAlegria - palhaços de hospital: proposta multidisciplinar de humanização em saúde. Rev Med (São Paulo). 2012;91(3):202-8.

6. Takahagui FM, Moraes ENS, Beraldi GH, Akamine GK, Basile MA, Scivoletto S. MadAlegria - estudantes de medicina atuando como Doutores-Palhaços: estratégia útil para a humanização do ensino médico? Rev Bras Educ Med. 2014;38(1):120-6.

7. Favoreto Jr CAOC. Narrative as a tool for the development of clinical practice. Interface Comunic Saude Educ. 2011;15(37):473-83.

8. Charon R. Narrative and medicine [perspective]. New Engl J Med. 2004;350(9):862-4.

9. Martins JJ, Schneider DG, Bunn KR, Goulart CA, Silva RM, Gama FO, Albuquerque GL. A percepção da equipe de saúde e do idoso hospitalizado em relação ao cuidado humanizado. Arq Catarinenses Med. 2008;37(1):30-7.

10. Beuter M, Alvim NAT. Expressões lúdicas no cuidado hospitalar sob a ótica de enfermeiras. Esc Anna Nery. 2010;14(3):567-574. doi: 10.1590/S141481452010000300019 .
11. Ford K, Courtney-Pratt H, Tesch L, Johnson C. More than just clowns - clown doctor rounds and their impact for children, families and staff. J Child Health Care. 2014;18(3):286-96. doi: 1177/1367493513490447.

12. Mussa C, Malerbi FTKM. O efeito do palhaço no estado emocional e nas queixas de dor de adultos hospitalizados. Psicol Rev (São Paulo). 2012;21:77-97.

13. Woodbury-Fariña MA, Antongiorgi JL. Humor. Psychiatry Clin North Am. 2014;37(4):561-78. doi: 10.1016/j. psc.2014.08.006.

14. Simons J. Hospital clowns boost healing through the power of laughter. Nurs Child Young People. 2015;27(2):15. doi: 10.7748/ncyp.27.2.15.s17.

15. Mota GM, Mota DMC, Machado MMT, Arrais RH, Oliveira CPV, Salgado MS, Souza MMB, William LH. A percepção dos estudantes de graduação sobre a atuação do "doutor palhaço" em um hospital universitário. Rev Bras Promoç Saúde. 2012;25(2):25-32. doi: 10.5020/18061230.2012.s25.

16. Campos-Brustelo TN, Bravo FF, Santos MA. Contando e encantando histórias de vida em um centro de atenção psicossocial. SMAD, Rev Eletrônica Saúde Mental Álcool Drog. 2010;6(1):1-11.

17. George DR, Stuckey HL, Whitehead MM. How a creative storytelling intervention can improve medical student attitude towards persons with dementia: a mixed methods study. Dementia (London). 2014;13(3):318-29. doi: $10.1177 / 1471301212468732$.

18. Pasche DF, Passos E, Hennington EA. Cinco anos da política nacional de humanização: trajetória de uma política pública. Ciênc Saúde Coletiva. 2011;16(11):45414548. doi: 10.1590/S1413-81232011001200027. 